Healthcare experience design: A conceptual and methodological framework for understanding the effects of usability on the access, delivery, and receipt of healthcare

Jessica Lynn Campbell

University of Central Florida, Orlando, FL, USA

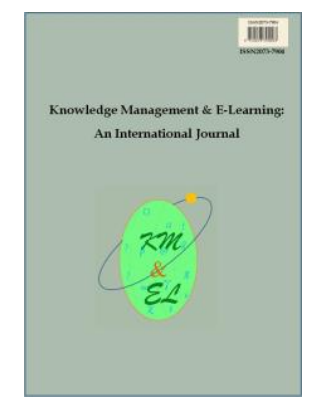

Knowledge Management \& E-Learning: An International Journal (KM\&EL) ISSN 2073-7904

Recommended citation:

Campbell, J. L. (2020). Healthcare experience design: A conceptual and methodological framework for understanding the effects of usability on the access, delivery, and receipt of healthcare. Knowledge Management \& E-Learning, 12(4), 505-520. https://doi.org/10.34105/j.kmel.2020.12.028 


\title{
Healthcare experience design: A conceptual and methodological framework for understanding the effects of usability on the access, delivery, and receipt of healthcare
}

\author{
Jessica Lynn Campbell* \\ Department of English \\ University of Central Florida, Orlando, FL, USA \\ E-mail: jessicalynn@embarqmail.com
}

*Corresponding author

\begin{abstract}
Health information technology (HIT) is a key component of healthcare today and has the potential to help support the quadruple aim of patient-centered healthcare. The access, delivery, and receipt of healthcare involves many stakeholders interacting with different HIT, and the usability of HIT is known to impact the acceptance and adoption of HIT, as well as health outcomes of populations. Understanding the influence that various individual and contextual factors have on the usability of HIT is challenging because of the network of interactions between humans and technology that occur in the same or different contexts within a unique healthcare system. Yet, it is important to identify usability problems in order to be able to design and implement HIT. This article begins by describing the current use of HIT with respect to the healthcare landscape. Next, usability is defined and demonstrated to play a critical role in users' acceptance and adoption of HIT. The remainder of the article describes a new framework called, healthcare experience design. The healthcare experience design framework models the healthcare experience, which is defined as the composite of all of an individual's interactions with other humans and HIT. The healthcare experience design framework is a useful conceptual model and methodological approach to identify usability problems in the healthcare system, and its practical application is illustrated using an artificial use case.
\end{abstract}

Keywords: Health information technology; HIT; Usability; Healthcare; Usability testing; Healthcare experience

Biographical notes: Jessica Lynn Campbell has a Master's in EnglishTechnical Communication and a $\mathrm{PhD}$ in Philosophy, from the University of Central Florida. Dr. Campbell is an expert and experienced technical communicator, author, and multi-media manager and has years of professional industry experience as a technical communicator composing multimedia content. Jessica teaches a variety of technical communication courses where she focuses on usability principles. Jessica's expertise is in evaluating the effects of the usability of health information technology (HIT) and communications and the user-experience, investigating HCI of HIT, and how to design for usability. Her scholarship includes both theoretical and practical applications. She has performed quantitative and qualitative research and encourages interdisciplinary and collaborative research. 


\section{Introduction}

Health information technology (HIT) is a critical component of healthcare today. HIT is used to deliver healthcare to patients in remote locations through telemedicine (Bashshur, 1995) and support health-related activities, such as home monitoring of patients and patient self-care (Whitten, 2006). Electronic Health Records (EHRs) and Clinical Decision Support Systems (CDSSs) integrate salient patient medical data on user interfaces and support clinicians during the diagnoses and treatment process (Horsky \& Ramelson, 2016; Li et al., 2012). In addition, consumers are increasingly using mobile health applications (mHealth) (Schneider et al., 2016) and online health information (eHealth) to access health information and become active participants in their own health (Eysenbach, 2005).

It is imperative that HIT be designed to be used effectively and safely by the wide range of users who may engage with these applications during the access, delivery, and receipt of healthcare (Borycki, 2015; Borycki \& Kushniruk, 2005). The usability of HIT is increasingly recognized as a key factor in physicians and patients' adoption of telemedicine, EHR, mHealth interventions, and other eHealth interventions that have the potential to improve health outcomes (Lazard et al., 2016; Monkman \& Kushniruk, 2013; Roman et al., 2017; Wilson \& Lankton, 2004). Therefore, examining the usability of HIT plays a vital role in ensuring that healthcare systems are effective, efficient, and usable by a broad range of stakeholders with different physiologic and psychologic capabilities. The potential for HIT to improve healthcare cannot be achieved if these technologies are neither designed nor implemented without understanding the relationships between usability with the overall user experience.

Healthcare is referred to as every aspect, service, actor, and technology involved in the care of one's health (Wax, 2019). Wax (2019) states, "it includes, but is not limited to: physician offices, hospitals, labs, radiology centers, physical therapy offices, pharmaceutical companies, pharmacies, and now health insurance companies, group purchasing organizations, pharmacy benefit managers, corporate healthcare systems, and combinations of insurance/PBM/pharmacy and much more." From a human factors perspective, the combined experience of any one actor involved in the access, delivery, and receipt of healthcare is referred to as the healthcare experience (ISO, 2010; Punchoojit \& Hongwarittorrn, 2017).

Additionally, a cultural shift of modern healthcare systems is towards a patientcentered healthcare paradigm, which has four key objectives:

- $\quad$ reducing healthcare costs,

- $\quad$ improving the patient experience,

- $\quad$ improving the physician experience, and

- $\quad$ improving the health of populations (Bodenheimer \& Sinsky, 2014; Millenson, 2014; Tuckson, Edmunds, \& Hodgkins, 2017).

Usable HIT plays a significant role in reaching the quadruple aim of patientcentered healthcare and supports the claim that the usability of HIT must be considered from the perspectives of all stakeholders involved in a healthcare situation. Furthermore, HIT can facilitate the continuity of care for patients when different physicians, nurses, caregivers, and patients may all use the same or similar HIT, such as EHRs, online patient portals, telemedicine, mHealth, and more computer-based health information. 
Furthermore, since the 1980s, the continuity of care has been regarded as a crucial component of quality healthcare and influences patient satisfaction, costs, and patient health outcomes (Alazri et al., 2007; Artnak et al., 2011; Gonnella \& Herman, 1980). The continuity of care is defined as the process (interactions between users and services) of delivering healthcare that evaluates patients' health status before and after care, uses appropriate measures of health outcomes, and considers all potential extrinsic factors that may impact a patient receiving quality healthcare and improved health outcomes (Alazri et al., 2007; Gonnella \& Herman, 1980). The continuity of care, thus, includes the interactions between patients and other stakeholders involved in their healthcare, as well as the technology that is used in this process.

Given that current HIT usability design and research lacks in consistency or standardization (Ellsworth, 2017; Yen \& Bakken, 2011), understanding the various factors that affect the usability of HIT is a challenging undertaking. There is a myriad of different applications of HIT that take place in different contexts and every healthcare situation is unique (St.Amant, 2019b). Identifying usability problems in a complex network of human and technology interactions requires a framework that accounts for each stakeholder in the system, the context of use, and the implications of usability during the interactions between humans and technology, which are often concurrent and shape one another (Jensen, 2015). Collectively, these interactions construct the entire healthcare experience.

A comprehensive conceptual and methodological approach to understanding the usability of HIT, by many stakeholders, in different contexts needs to encompass this system of human-computer interactions (HCIs) holistically. There are the patients to consider and their perspectives; there are the physicians to consider and their perspectives; there are caregivers to consider and their perspectives; and there are the numerous interactions with each other and multiple touchpoints with technology, often with disparate systems, that impact usability. Yet, collectively, these components bricolage the healthcare experience. Moreover, usability is moderated by many socio-cognitivetechnical factors, such as eHealth literacy (Kushniruk et al., 1997; Mackert et al., 2009; Mackert et al., 2016; Monkman \& Kushniruk, 2015), demographics (George et al., 2012; Wilson, 2009), and an individual's decision-making process (Kushniruk, 2001). Thus, there is a need for a broader understanding of how to design HIT usability studies, perform usability testing, and ultimately design HIT that mitigate disparities in healthcare and health outcomes rather than augment.

This exploration of the healthcare experience can be conceptualized by a novel framework, termed, healthcare experience design. This framework offers a fresh perspective on the healthcare experience by drawing knowledge from several disciplines and theories. Given the depth and breadth in which HIT is employed and consumed in the healthcare system and in the management of one's health, a model that depicts these unique healthcare scenarios needs to account for all stakeholders, technology, and activities that occur in the access, delivery, and receipt of healthcare.

The purpose of this article is to describe the healthcare experience design framework and demonstrate how it is a useful conceptual model to understand the usability of HIT and its impact on the healthcare experience. In addition, healthcare experience design is a useful methodological tool for scholars and practitioners to be able to design usability studies situated in the healthcare experience by mapping their subjects and units of analysis in the architecture of the healthcare experience design framework. An artificial use case scenario will be used to demonstrate how healthcare experience design can be applied in the study of the usability of a telemedicine platform. 


\section{Background: Concepts, theories, and methods}

\subsection{Usability}

Usability is defined by the ISO, as the extent to which a product can be used by target users to achieve specific goals with effectiveness, efficiency, and satisfaction in their context of use (ISO, 2018; Yen \& Bakken, 2012). The relationship between usability, quality of healthcare, and patient safety is apparent. Poor usability of HIT, like Computerized Physician Order Entry (CPOE) systems, EHRs, and CDSSs can result in inefficiency, poor workflow and communication between clinicians and nurses, increased cognitive workload, inaccurate diagnoses, medication dispensing error, and low user satisfaction (Beuscart-Zéphir et al., 2004; Horsky \& Ramelson, 2016; Kaipio et al., 2020; Kastner et al., 2010; Kushniruk, 2001). Insufficient or poor usability of HIT, in all of these cases, can compromise patient safety and is one leading cause of the lack of adoption of specific HIT that are intended to increase access and quality of healthcare (Goldberg et al., 2011; Turner et al., 2017).

Numerous studies demonstrate the various socio-cognitive-technical and cultural factors that influence any one individual's usability of HIT (Fig. 1). These are often called the human factors of usability and are both subjective and contextual (Borycki, 2015; Carayon \& Hoonakker, 2019; Gibbons et al., 2014; Punchoojit \& Hongwarittorrn, 2017).

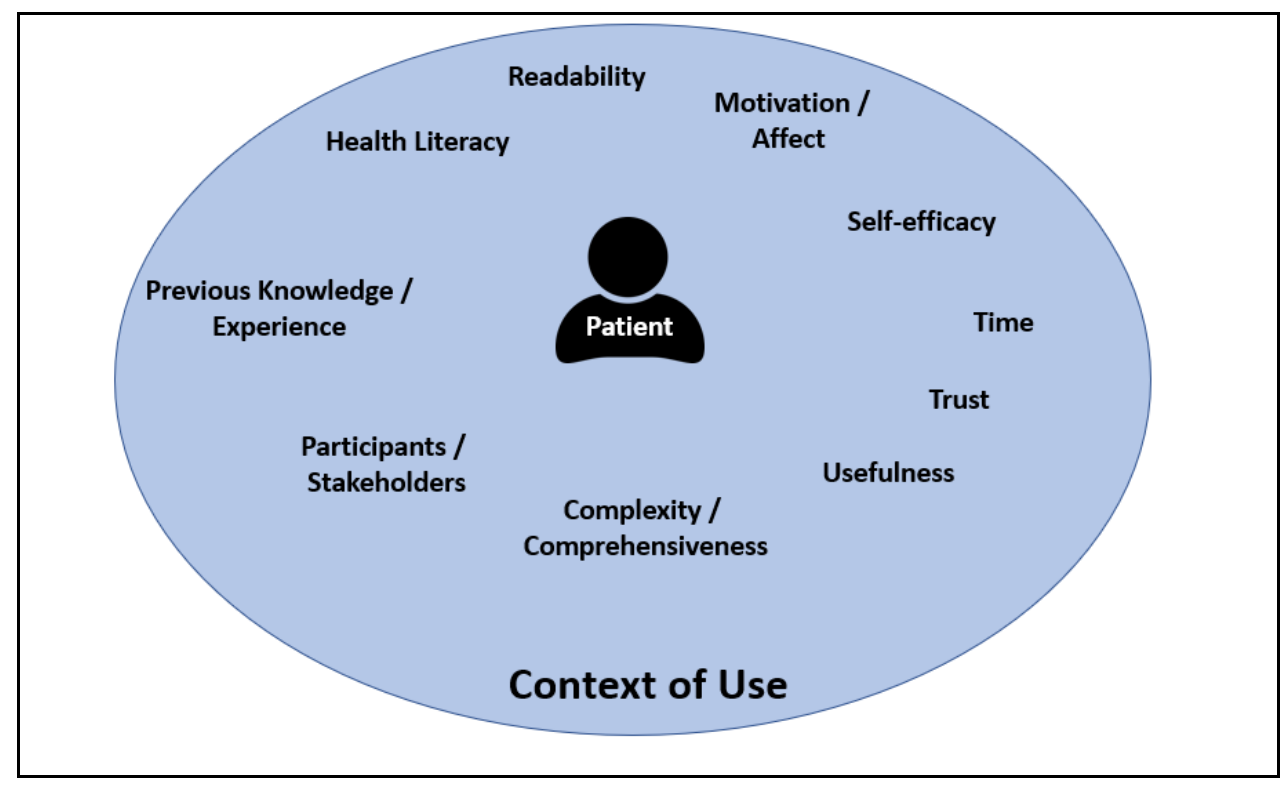

Fig. 1. A patient situated in a context interacting with HIT. The human factors affecting the usability of HIT are demonstrated to impact the patient in the context of the interaction.

For instance, subjective aspects, such as a user's cognitive processing capabilities or age (Chaudhrya et al., 2019; Kushniruk, 2001; Lin et al., 2009), as well as contextual determinants, including one's geographic location, organizational practices, and even time to perform a task (Giordanengo et al., 2019; Peddle, 2007; Sandefer et al., 2015) all affect the usability of HIT. Design flaws in a user interface, which is the point of 
interaction between a user and a HIT, are known to generate usability problems or make usability issues more pronounced (Gibbons et al., 2014; Or \& Tao, 2012). Consequently, poor usability or insufficient meaningful use of HIT may increase disparities in the healthcare system.

Although usability is an imperative in the design and study of HIT, the criteria to achieve usability are often quantified differently or evaluated using different metrics. For instance, the criteria used to gauge usability can stem from the definition of usability: effectiveness, efficiency, and satisfaction (Rogers et al., 2007). Kushniruk and colleagues (Borycki \& Kushniruk, 2005; Kushniruk et al., 2010; Kushniruk et al., 2013) underscore the need for safety in the testing of HIT. Punchoojit and Hongwarittorrn (2017) suggest that some usability criteria can hardly be measured, such as satisfaction and engagement, because these constructs are subjective and involve human emotion. Other researchers argue that a conceptual framework to understanding the impact of the usability of HIT must address the following four dimensions: technology platform, technology functionality, information content, and the user interface (Gibbons et al., 2014).

This challenge in understanding usability and how to evaluate usability in the context of the healthcare system needs to be met with a holistic conceptual and methodological approach that allows researchers to identify actors, define usability constructs, and employ the best methods to evaluate and measure usability. Yet, despite the various metrics of usability, subjective constructs, and features of HIT that have been studied and identified to impact the healthcare experience, they all reveal the role that HIT and humans interacting with each other plays in the journey to achieve positive health outcomes. The ultimate goal of healthcare - to achieve positive health outcomescannot be overstated and is indubitably a universal goal for all actors and technology involved in the access, delivery, and receipt of healthcare. Therefore, I suggest a new framework (healthcare experience design) for outlining this healthcare experience journey to achieve positive health outcomes.

\subsection{Technical communication}

There are numerous theories and methods used to evaluate the usability of HIT, gain insight on patient satisfaction, and assess the utilization and efficacy of HIT. Patient experience design (PXD) is the term developed by Meloncon (2017) characterizing the, "participatory methodological approach centered on contextual inquiry to understand the relationship between information (or technology) and human activities in healthcare" ( $p$. 20). The concept of health literacy - and eHealth literacy - is imperative for the safe and effective use of HIT and can also increase social disparities in healthcare and the health of particular populations who are not able to access or use HIT due to insufficient or poor health literacy (Monkman \& Kushniruk, 2013, 2015; Sarkar et al., 2010). Technical communicators are incumbent in the design of HIT because the discipline is usercentered and has the expertise to compose, design, and deliver information that meets the needs of the target user group.

This interplay between technical communication and the user is also expressed by the overlap between technical communication and the emphasis on context (Redish, 2007; Meloncon, 2016; St.Amant, 2017, 2019b). Healthcare does not occur in a silo, it can occur in many different contexts. The context of use is more than just the physical location of users when they encounter a health situation, it also includes other individuals in their environment, the medium of healthcare delivery, their goals for use, as well as how they are able to use HIT to achieve positive health outcomes. These factors shape the 
healthcare experience. Technical communicators are primed to design and delivery complex health information and maximize user interfaces for as many users as possible (St.Amant, 2019a).

\subsection{Activity theory}

Context of use is germane to activity theory. Activity theory is a socio-cognitive approach to understanding human activities as phenomena situated in a social system (Vygotsky, 1978; Leont'ev, 1978). Activity theory is popular in the Human-Computer Interaction (HCI) community because it primarily focuses on how humans use tools - or technology - during activities that are performed to achieve certain goals (Kuutti, 1996; Nardi, 1996; Wilson, 2008). Activity theory is useful in the context of a healthcare situation because it attempts to model the interactions between users, called subjects, tools, which are the computer-based systems or digital health applications, and the interactions between the two, which is the activity or sequence of activities performed. The activity theory model, often called the activity system, is composed of several components, and is often represented by a triangle illustrating how users interact with tools in order to achieve specific goals (Fig. 2). These actions, described by Vygotsky (1978), are "mediated acts." The act of mediation indicates how subjects or agents make conscious decisions to use objects or tools in ways that support their activity, yet the design and functionality of as tool inherently shapes an individual's successful use of the tool. Leont'ev (1978) expanded on the concept of mediation by adding a hierarchical system consisting of a socio-technical system whereby individual activities constitute the execution of one main operation. This is a useful conception when considering all of the different actors (e.g., administrators, doctors, nurses, patients, caregivers) and activities (complete in-take forms, physical examination, engage with EHR, provide patient education, shared-decision making) involved in the practice of healthcare. In the case of a healthcare situation, various users interact with HIT in different contexts, yet all activities are performed in order to achieve positive health outcomes.

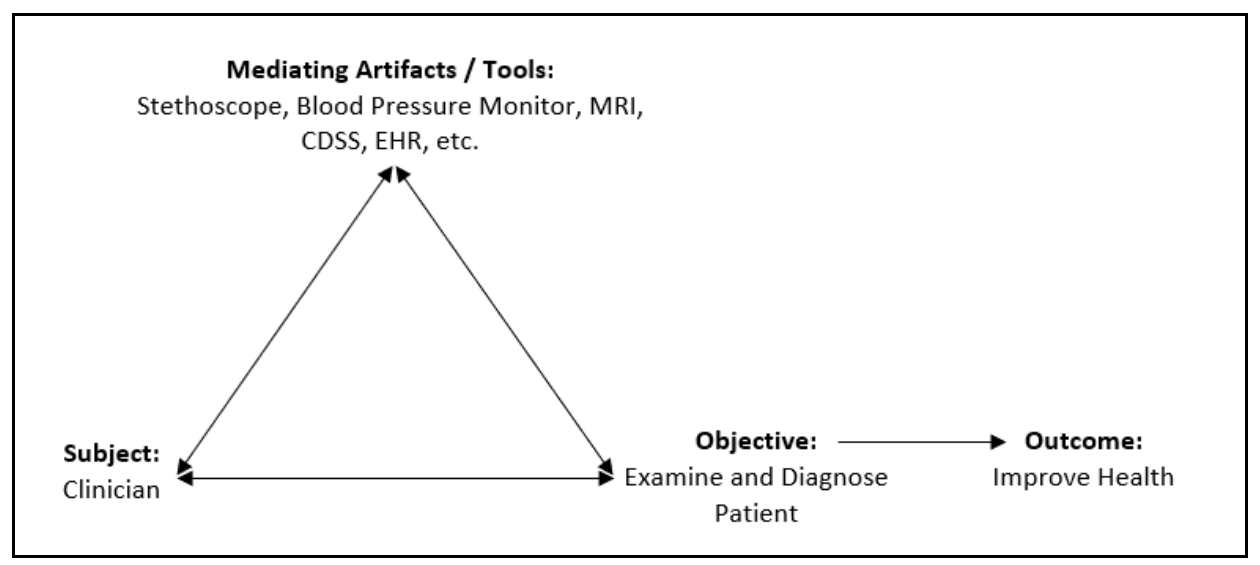

Fig. 2. An example of a healthcare activity system demonstrating the interaction between a subject and tools directed towards an objective. The final outcome is to achieve positive health outcomes.

Activity theory also contributes the notion of "contradictions" in the activity system (Fig. 3). 


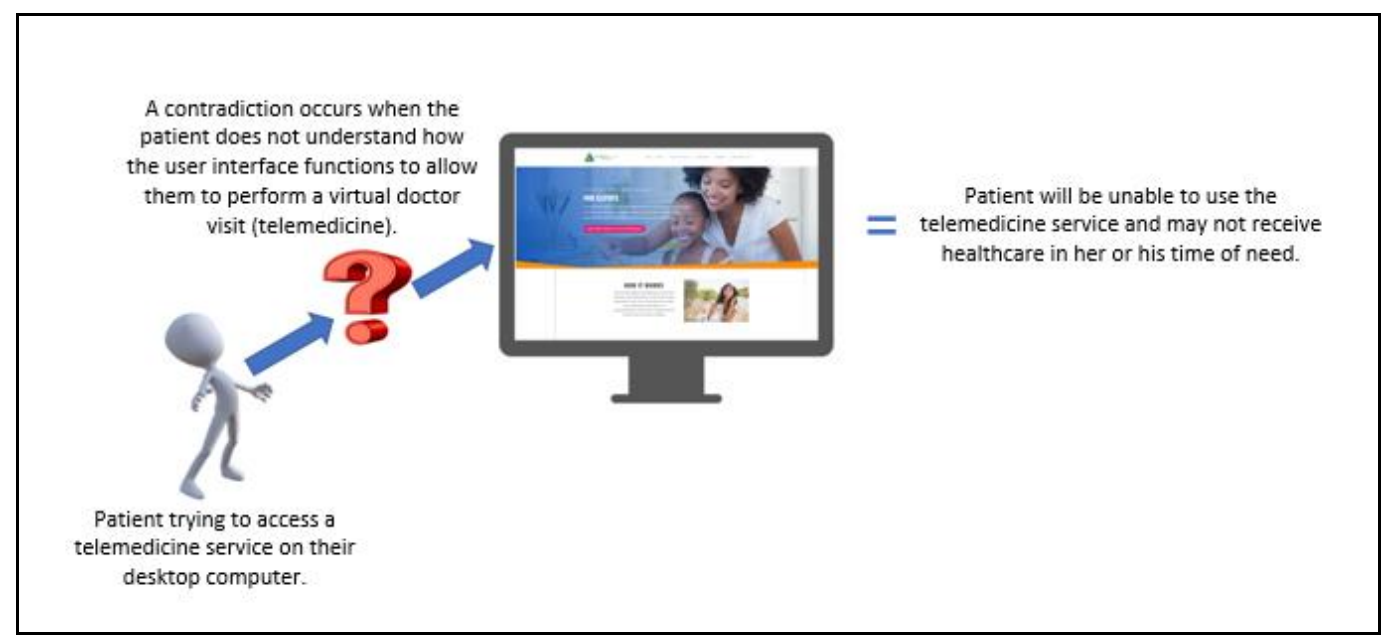

Fig. 3. Contradictions during the interaction between a patient and her or his meaningful use of a HIT

Given that activities are constantly evolving and altered, contradictions appear in an activity system when there are tensions between an activity and any other construct in the activity system or with other activity systems (Engeström, 1987; Sadeghi et al., 2014; Wiser et al., 2017). Contradictions can result when users' do not understand how a technology or system functions or how to interact with it effectively to support the activity they are performing. Contradictions, essentially, bring to surface usability problems resulting from poor user interface design. For instance, if a clinician must enter patient medical data in an EHR, and the EHR user interface does not include a specific medical code in a menu, this is a contradiction between how the clinician needs to use the EHR and how the EHR is designed. Contradictions are crucial for identifying usability problems that are embedded in the user interface (Spinuzzi, 1999; Vrazalic, 2003).

Activity theory draws knowledge from several disciplines, including cognitive psychology, HCI, anthropology, philosophy, and linguistics (Patel \& Kushniruk, 1998), and it is useful as a descriptive tool to understand humans' cognitive processes as they interact with objects in relationship to other socio-technical constructs (Engeström, 2000; Nardi, 1996; Patel \& Kushniruk, 1998).

\subsection{User-centered design \& usability inspection methods}

Both the technical communication and HCI disciplines draw concepts from cognitive science and sociology to understand and design health information that aims for usability. Successful results can be achieved through a series of steps involving analyzing users' needs, developing prototypes of a HIT, and performing usability testing with representative users interacting with these prototypes as they perform real-world tasks (Norman \& Draper, 1986). Iterative design is an important step in this process, which is the design modifications done to the technology after the results from usability testing have revealed usability problems (Borycki \& Kushniruk, 2005; Kushniruk, 2015; Kushniruk \& Patel, 2004; Li et al., 2012). This process is called, user-centered design, by the technical communication community (Redish, 2007; Spinuzzi, 1999; St.Amant, 2017), and involves a collection of different methods under the title, usability engineering 
methods, by HCI experts (Monkman \& Kushniruk, 2015; Kushniruk \& Patel, 2004; Kushniruk \& Patel, 2005; Nielsen, 1993).

Regardless of which camp one identifies with, user-centered design and usability inspection methods both are intended to achieve optimal usability for a target user group (Goldberg et al., 2011). Combined with the goal of designing for UX, “...to encourage positive feelings (e.g., satisfying, enjoyable, exciting, motivating, and fun) and minimizing negative feelings (e.g., boring, frustrating, annoying, and cutesy) towards the product" (Punchoojit \& Hongwarittorrn, 2017), the healthcare experience design framework encompasses the holistic nature of the access, delivery, and receipt of healthcare in order to be able to identify the qualities of usability and a user's perspective that impact the healthcare experience.

\section{Healthcare experience design}

\subsection{What is healthcare experience design?}

Healthcare experience design is a conceptual and methodological tool that represents the healthcare experience of any one subject as they progress through the healthcare system. It is a composite of all of an individual's interactions with other humans, from physicians to healthcare insurance providers, and HIT, from web-based patient portals, EHRs, mHealth applications, to other HIT. It is important to understand the network of interactions that occur when designing and implementing HIT because the usability of HIT from any one subject's perspective will inherently impact the usability of that same HIT by another subject (Kaipio et al., 2020; Kastner et al., 2010; Powell et al., 2017; Wang et al., 2019).

Contradictions or breakdowns in the healthcare system accentuate usability problems. Once identifying usability problems in one component of the system, the effects on the relationship between usability and other interactions in the system are also able to be identified, which consequently impact the healthcare experience. For instance, in Kaipio et al's (2020) study of the usability of EHRs from nurses and physicians' viewpoints, nurses found minor technical problems with the user interface, like loss of documented information, to be unsatisfying and result in inefficiencies, whereas, physicians found the EHR easy-to-use. Similarly, Powell et al's (2017) study of patients' perceptions of telemedicine visits revealed that some patients felt the emotional loss of the face-to-face visit with a physician, while other patients using the same telemedicine service felt comfortable with the level of engagement with the physician. Additionally, caregivers' remarks were favorable towards being able to be present during a telemedicine visit whereas they would not have been able to be present at an in-office visit. In another example, Monkman and Kushniruk (2013) performed usability testing of a Personal Health Record (PHR) and discovered that users with limited eHealth literacy found the lack of a legend on the blood pressure graph and medication terminology difficult to understand and use to make decisions about their health. An individuals' eHealth literacy level, "would undoubtedly create problems for users' ability to understand and make use of their health information," (Monkman \& Kushniruk, 2015), and must be considered when designing and delivering HIT.

The examples provided elucidate how socio-cognitive-technical and cultural factors affect the usability of HIT and affect different actors in different ways. However, all of these dynamic interactions impact the healthcare experience because the ultimate 
goal of healthcare and the use of HIT is to achieve positive health outcomes. The healthcare experience design framework is a useful and valuable tool to map out the access, delivery, and receipt of healthcare from any subject's perspective in order to be able to identify where usability problems may erupt and affect health outcomes.

\subsection{A holistic approach to identifying the interactions between humans and technology in a healthcare network}

Because usability problems can erupt from a user's interaction with a poorly designed HIT to the same degree that an individual's cognitive capabilities and context of use can impact their ability to meaningfully interact with a HIT to achieve positive health outcomes, the healthcare experience design framework can be used as a conceptual model to be able to identify these components of the healthcare experience. Researchers can use the healthcare experience framework to map out the journey of any one subject or actor in the healthcare system and be able to design studies that are valuable to identifying the effects of usability on the healthcare experience.

Healthcare experience design takes concepts from several theories and framework. Healthcare experience design, as shown in Fig. 4, recognizes that various agents in the healthcare system, interact with each other and with technology, and these interactions are situated in a context. The overarching goal for all the activities in the healthcare network is to improve the health of patients (achieve positive health outcomes). The interactions between agents or users, are individual activity components in the healthcare system. Each activity component may overlap with another. Usability problems that occur at the individual level may contribute to poor usability of the same or different HIT during another agent's interaction.

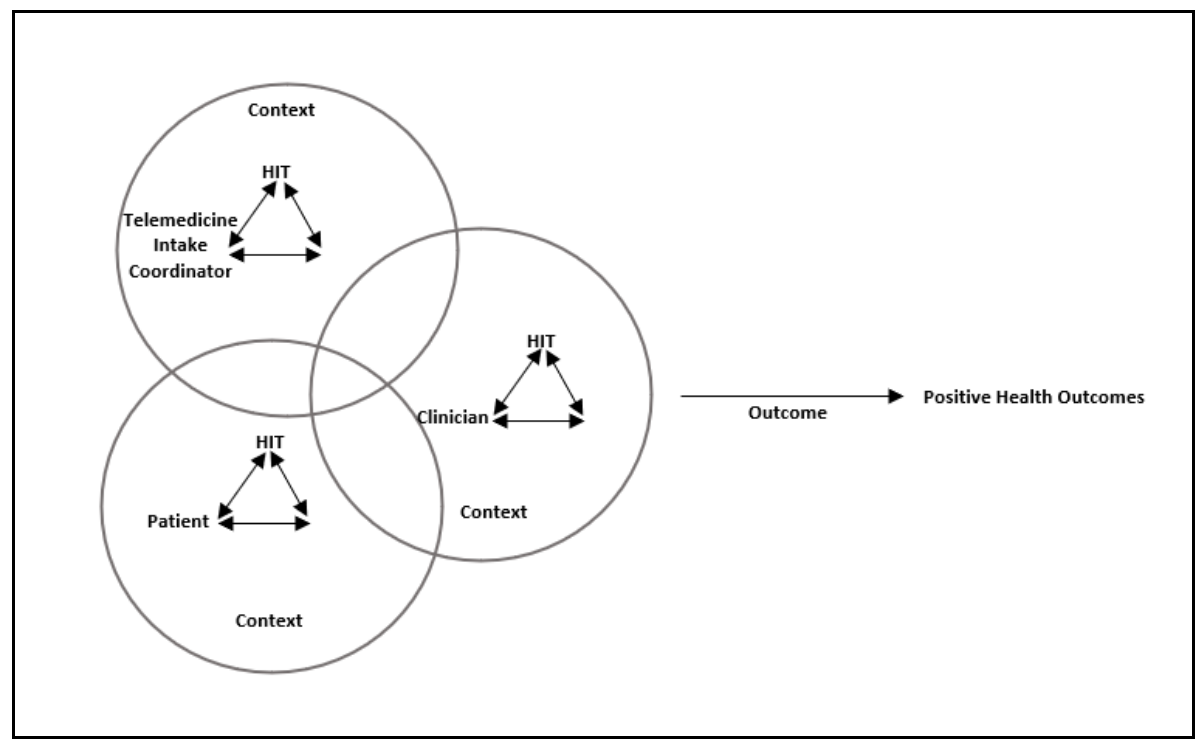

Fig. 4. Example of the healthcare experience design framework. A patient, intake coordinator, and clinician all employ the same telemedicine platform to provide healthcare. The healthcare experience design framework expresses where individual activity components overlap and the relationship between usability. 
Once identifying the usability problems exposed by contradictions that exist with one component in the healthcare experience, it can be plugged into the entire healthcare experience network to be able to discover the interplay between individual components and the relationship with usability, as well as the entire healthcare experience. Healthcare experience design situates the subject or human in the context of their interaction with a HIT and situates this component within a network of interactions with other subjects and HIT.

This new framework does not discount existing models (PXD (Meloncon, 2017), eHealth literacy framework (Norgaard et al., 2015), TURF (Zhang \& Waljiac, 2011), but rather expands on these models by merging concepts and theories from technical communication, HCI, and other socio-cognitive perspectives to address the entire healthcare experience. Insight gained from employing this conceptual model or methodology should inform the design, development, implementation, and use of tools to ensure all stakeholders involved in a healthcare experience receive beneficial outcomes (Kayser et al., 2015; Kushniruk, 2019).

Next, an example is provided regarding how the healthcare experience design framework can be used to conceptualize a patient moving through the healthcare system in order to achieve positive health outcomes. By using this model, one is able to visualize all of the various interactions between humans and technology that occur and identify areas where usability problems may be pronounced.

\subsection{Healthcare experience design: A use case scenario}

Healthcare experience design is a useful and valuable conceptual model and methodological approach. Next, an artificial use case scenario (Fig. 5) is provided to demonstrate how healthcare experience design can be applied in the study of the usability of a various HITs involved in any unique healthcare experience.

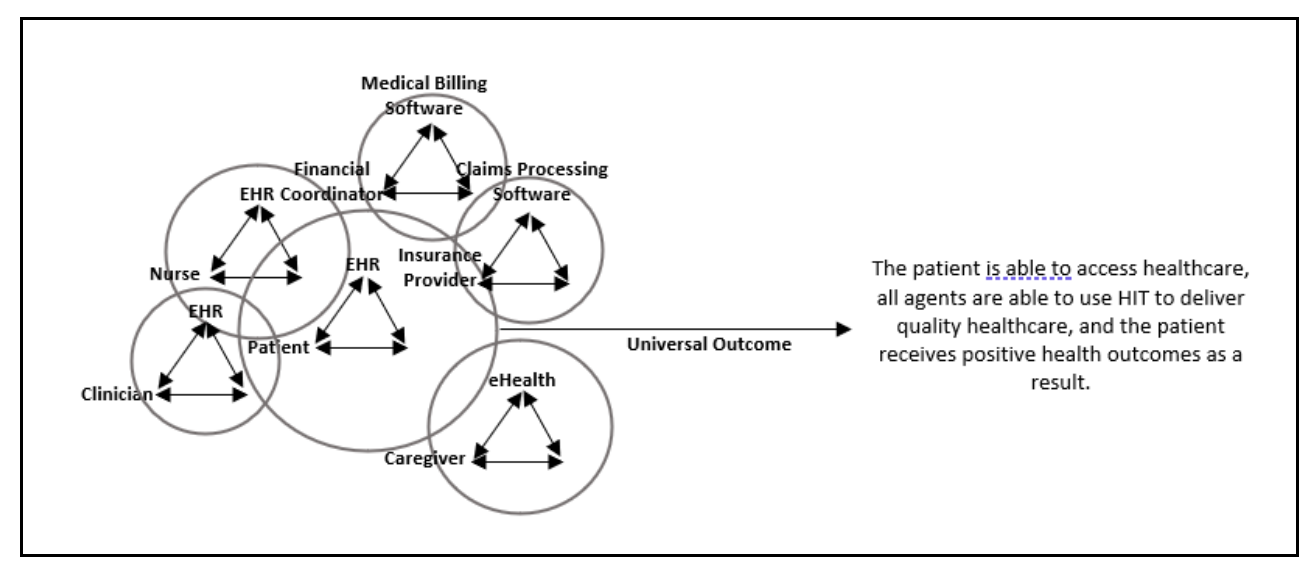

Fig. 5. Artificial use case scenario representing the overlapping interactions and contexts of a healthcare experience. A clinician, nurse, and patient all may use an EHR, in the same and different contexts; a financial coordinator, an insurance provider, and patient may all interact in the same and different contexts using different HIT; a caregiver may also be involved during a patient's healthcare before, during, and after the delivery of

healthcare, and thus may overlap other activity components in similar contexts.

A researcher who wanted to evaluate the usability of a particular EHR can use healthcare experience design to map out the healthcare network that would feature the 
EHR under investigation. As shown in the artificial use case in Fig. 5, the healthcare experience design framework allows researchers to be able to identify the various agents or users who interact with the EHR. Thus, the usability from the perspective of these users would need to be accounted for and researchers are able to select the best representative users to perform usability testing of the EHR. Furthermore, the healthcare experience design framework is also able to express the context in which humans and HIT interactions take place allowing researchers to perform usability testing in environments that closely resemble the real-life context of use. Usability is influenced by many individual and contextual factors, and experts argue that usability testing of HIT should be conducted in contexts that simulate a real-life situation and that are naturalistic - in the actual context of use, if possible (Borycki \& Kushniruk, 2005; Borycki et al., 2015; Kushniruk et al., 2013). Healthcare experience design is an approach to carefully designing usability studies that best mimic a real-life healthcare experience.

The healthcare experience network includes:

- $\quad$ Context - The physical location, time, space, and social context in which the subject performs the activity.

- $\quad$ Subject / Agent / User - The individual performing actions and tasks, making conscious decisions.

- Mediated artifact / Tool - The health information technology or other nonmaterial artifact that mediate the interactions between humans and other humans and human's goal-directed activities.

The main objective of the healthcare experience is to achieve positive health outcomes. Once identifying these parts of the healthcare experience, researchers and practitioners can begin to map out how each context, subject, and tool overlap, and then discover areas where contradictions are likely to occur.

The healthcare experience design framework is intended to be used flexibly because every user and every healthcare situation is unique and can take place in different contexts. In addition, because usability is influenced by so many fluctuating factorssocio-cognitive-technical and cultural influencers, a flexible approach is required in order to be able to accommodate the dynamic nature of healthcare and adapt to the circumstances of one's study.

\section{Conclusion}

Despite being new, the healthcare experience design framework blends existing theories and concepts from technical communication, HCI, and cognitive science by applying it to the understanding and testing of usability of HIT. Healthcare experience design can be used as a conceptual model and affords designers and health information providers a useful visualization of the healthcare experience in order to approach designing HIT to be used by target audiences in efficacious and safe ways. In addition, when designing usability studies, healthcare experience design can be used as a methodological approach to map out the access, delivery, and receipt of healthcare, isolate any one component in the healthcare system, and be able to understand the relationship between humans, technology, and usability, as well as how this impacts health outcomes. When designing studies, it is important to perform usability testing with representative target users, in their context of use, and define the constructs of usability that are being measured, such as the user's satisfaction or user interface display suitability. The healthcare experience 
design framework is flexible because it allows researchers and practitioners to collaborate and plug into the framework the actors, context, and other elements depending on their expertise, the unique healthcare situation, the HIT, the usability metrics they want to study, and the resources they have available.

Usability is a significant factor in the critical design and evaluation of HIT that is to receive wide-spread consumer adoption and that is to be used safely and effectively. Given that every healthcare situation is unique and the various and dynamic individual and contextual determinants of usability, healthcare experience design is expected to gain attention and traction quicky because of its implications for researchers and practitioners. All individuals have the opportunity to be positively impacted by HIT that is designed for usability, which can be facilitated by the healthcare experience design framework.

\section{Author Statement}

The authors declare that they have no conflict of interest.

\section{ORCIID}

Jessica Lynn Campbell (iD https://orcid.org/0000-0003-0728-3560

\section{References}

Alazri, M., Heywood, P., Neal, R. D., \& Leese, B. (2007). Continuity of care: Literature review and implications. Sultan Qaboos University Medical Journal, 7(3), 197-206.

Artnak, K. E., McGraw, R. M., \& Stanley, V. F. (2011). Health care accessibility for chronic illness management and end-of-life care: A view from rural America. Journal of Law, Medicine \& Ethics, 39(2), 140-155.

Bashshur, R. L. (1995). On the definition and evaluation of telemedicine. Telemedicine Journal (continued by Telemedicine and e-Health), 1(1), 19-30.

Beuscart-Zéphir, M. C., Pelayo, S., Degoulet, P., Anceaux, F., Guerlinger, S., \& Meaux, J. J. (2004). A usability study of CPOE's medication administration functions: Impact on physician-nurse cooperation. Studies in Health Technology and Informatics, 107(Pt 2), 1018-1022.

Bodenheimer, T., \& Sinsky, C. (2014). From triple to quadruple aim: Care of the patient requires care of the provider. The Annals of Family Medicine, 12(6), 573-576.

Borycki, E. M. (2015). Towards a framework for teaching about information technology risk in health care: Simulating threats to health data and patient safety. Knowledge Management \& E-Learning, 7(3), 480-488.

Borycki, E., \& Kushniruk, A. (2005). Identifying and preventing technology-induced error using simulations: Application of usability engineering techniques. Healthcare Quarterly, 8(SI), 99-105.

Borycki, E. M., Kushniruk, A. W., Kuziemsky, C. E., \& Nøhr, C. (Eds.) (2015). Context sensitive health informatics: Many places, many users, many context, many uses. Fairfax, VA: IOS Press, Inc.

Carayon, P., \& Hoonakker, P. (2019). Human factors and usability for health information technology: Old and new challenges. Yearbook of Medical Informatics, 28(1), 71-77.

Chaudhrya, A. P., Samudrala, S., Lopez-Jimenez, F., Shellum, J. L., Nishimura, R. A., Chaudhry, R., . . . Arruda-Olson, A. M. (2019). Provider survey on automated clinical decision support for cardiovascular risk assessment. Mayo Clinic Proceedings: 
Innovations, Quality \& Outcomes, 3(1), 23-29.

Ellsworth, M. A., Dziadzko, M., O’Horo, J. C., Farrell, A. M., Zhang, J., \& Herasevich, V. (2017). An appraisal of published usability evaluations of electronic health records via systematic review. Journal of the American Medical Informatics Association, 24(1), 218-226.

Engeström, Y. (1987). Learning by expanding: An activity theoretical approach to developmental research. Helsinki, Finland: Orienta-Konsultit.

Engeström, Y. (2000). Activity theory as a framework for analyzing and redesigning work. Ergonomics, 43(7), 960-974.

Eysenbach, G. (2005). Design and evaluation of consumer health information web sites. In D. Lewis, G. Eysenbach, R. Kukafka, P. Z. Stavri, \& H. B. Jimison (Eds.), Consumer Health Informatics (pp. 34-60). New York, NY: Springer.

George, S., Hamilton, A., Baker, R. S. (2012). How do low-income urban African Americans and Latinos feel about telemedicine? A diffusion of innovation analysis. International Journal of Telemedicine and Applications. doi: 10.1155/2012/715194

Gibbons, M. C., Lowry, S. Z., \& Patterson, E. S. (2014). Applying human factors principles to mitigate usability issues related to embedded assumptions in health information technology design. JMIR Human Factors, 1(1), e3.

Giordanengo, A., Årsand, E., Grøttland, A., Bradway, M., \& Hartvigsen, G. (2019). Acceptance barriers of using patients' self-collected health data during medical consultation. In Proceedings of the 17th Scandinavian Conference on Health Informatics (pp. 50-55). Oslo Norway.

Goldberg, L., Lide, B., Lowry, S., Massett, H. A., O’Connell, T., Preece, J., Quesenbery, W., \& Shneiderman, B. (2011). Usability and accessibility in consumer health informatics: Current trends and future challenges. American Journal of Preventive Medicine, 40(5), S187-S197.

Gonnella, J. S., \& Herman, M. W. (1980). Continuity of care. JAMA, 243(4), 352-354.

Horsky, J., \& Ramelson, H. Z. (2016). Development of a cognitive framework of patient record summary review in the formative phase of user-centered design. Journal of Biomedical Information, 64, 147-157.

International Organization of Standardization (ISO), 9241-210. (2010). Ergonomics of human-system interaction-part 210: Human-centred design for interactive systems. Retrieved from https://www.iso.org/standard/52075.html

International Organization for Standardization (ISO), 9241-11. (2018). Ergonomics of human-system interaction -- Part 11: Usability: Definitions and concepts (2nd ed.). Switzerland: ISO/IEC.

Jensen, S. (2015). Patient safety and quality of care: How may clinical simulation contribute? Knowledge Management \& E-Learning, 7(3), 412-424.

Kaipio, J., Kuusist, A., Hyppönen, H., Heponiemi, T., \& Lääveri, T. (2020). Physicians' and nurses' experiences on EHR usability: Comparison between the professional groups by employment sector and system brand. International Journal of Medical Informatics, 134: 104018.

Kastner, M., Lottridge, D., Marquez, C., Newton, D., \& Straus, S. E. (2010). Usability evaluation of a clinical decision support tool for osteoporosis disease management. Implementation Science, 5: 96.

Kayser, L., Kushniruk, A., Osborne, R. H., Norgaard, O., \& Turner, P. (2015). Enhancing the effectiveness of consumer-focused health information technology systems through eHealth literacy: A framework for understanding users' needs. JMIR Human Factors, 2(1): e9.

Kushniruk A. (2019). The importance of health information on the Internet: How it saved my life and how it can save yours. Journal of Medical Internet Research, 21(10): 
e16690.

Kushniruk, A., Beuscart-Zéphir, M.-C., Grzes, A., Borycki, E., Watbled, L., \& Kannry, J. K. (2010). Increasing the safety of healthcare information systems through improved procurement: Toward a framework for selection of safe healthcare systems. Healthcare Quarterly, 13(SI), 53-58.

Kushniruk, A., Nohr, C., Jensen, S., \& Borycki, E. M. (2013). From usability testing to clinical simulations: Bringing context into the design and evaluation of usable and safe health information technologies. Yearbook of Medical Informatics, 8(1), 78-85.

Kushniruk, A. W. (2001). Analysis of complex decision-making processes in health care: Cognitive approaches to health informatics. Journal of Biomedical Informatics, 34(5), 365-376.

Kushniruk, A. W. (2015). eHealth literacy: Emergence of a new concept for creating, evaluating and understanding online health resources for the public. Knowledge Management \& E-Learning, 7(4), 518-521.

Kushniruk, A. W., \& Patel, V. L. (2004). Cognitive and usability engineering methods for the evaluation of clinical information systems. Journal of Biomedical Informatics, $37(1), 56-76$.

Kushniruk, A. W., \& Patel, V. L. (2005). Cognitive approaches to the evaluation of healthcare information systems. In J. G. Anderson \& C. E. Aydin (Eds.), Evaluating the Organizational Impact of Healthcare Information Systems (pp. 144-173). New York, NY: Springer.

Kushniruk, A. W., Patel, V. L., \& Cimino, J. J. (1997). Usability testing in medical informatics: Cognitive approaches to evaluation of information systems and user interfaces. In Proceedings of Conference of the American Medical Informatics Association: AMIA Fall Symposium (pp. 218-222).

Kuutti, K. (1996). Activity theory as a potential framework for human-computer interaction research. In B. A. Nardi (Ed.), Context and Consciousness: Activity Theory and Human-Computer Interaction (pp. 17-44). Cambridge, MA: MIT Press.

Lazard, A. J., Watkins, I., Mackert, M. S., Xie, B., Stephens, K. K., \& Shalev, H. (2016). Design simplicity influences patient portal use: The role of aesthetic evaluations for technology acceptance. Journal of the American Medical Informatics Association (JAMIA), 23(e1), e157-e161.

Leont'ev, A. N. (1978). Activity, consciousness, and personality. Englewood, CO: Prentice Hall.

Li, A. C., Kannry, J., Kushniruk, A., Chrimes, D., McGinn, T. G., Edonyabo, D., ... Mann D. M. (2012). Integrating usability testing and think-aloud protocol analysis with "near-live" clinical simulations in evaluating clinical decision support. International Journal of Medical Informatics, 81(11), 761-772.

Lin, C. A., Neafsey, P. J., \& Strickler, Z. (2009). Usability testing by older adults of a computer-mediated health communication program. Journal of Health Communication, 14(2), 102-118.

Mackert, M., Kahlor, L., Tyler, D., \& Gustafson, J. (2009). Designing e-health interventions for low-health-literate culturally diverse parents: Addressing the obesity epidemic. Telemedicine and e-Health, 15(7), 672-677.

Mackert, M., Mabry-Flynn, A., Champlin, S., Donvan, E. E., \& Poundres, K. (2016). Health literacy and health information technology adoption: The potential for a new digital divide. Journal of Medical Internet Research, 18(10): e264. doi: 10.2196/jmir.6349

Meloncon, L. (2016). Patient experience design: Technical communication's role in patient health information and education. Intercom, 62(2), 12-16.

Meloncon, L. K. (2017). Patient experience design: Expanding usability methodologies for healthcare. Communication Design Quarterly, 5(2), 19-28. 
Millenson, M. L. (2014). Paradigm, not pill: The new role of patient-centered care. NIHCM Foundation. Retrieved from https://millenson.com/wpcontent/uploads/2013/03/Patient-Centered_Care_EV_Millenson_2014.pdf

Monkman, H., \& Kushniruk, A. (2013). Applying usability methods to identify health literacy issues: An example using a personal health record. Studies in Health Technology and Informatics, 183, 179-185. doi: 10.3233/978-1-61499-203-5-179

Monkman, H., \& Kushniruk, A. W. (2015). eHealth literacy issues, constructs, models, and methods for health information technology design and evaluation. Knowledge Management \& E-Learning, 7(4), 541-549.

Nardi, B. A. (1996). Context and consciousness: Activity theory and human-computer interaction. Cambridge, MA: The MIT Press.

Nielsen, J. (1993). Usability engineering. New York, NY: Academic Press.

Norgaard, O., Furstrand, D., Klokker, L., Karnoe, A., Batterham, R., Kayser, L., \& Osborne, R. H. (2015). The e-health literacy framework: A conceptual framework for characterizing e-health users and their interaction with e-health systems. Knowledge Management \& E-Learning, 7(4), 522-540.

Norman, D., \& Draper, S. (1986). User centered system design: New perspectives on human-computer interaction. Hillsdale, NJ: L. Erlbaum Associates Inc.

Or, C., \& Tao, D. (2012). Usability study of a computer-based self-management system for older adults with chronic diseases. Journal of Medical Internet Research (JMIR) Research Protocols, 1(2): e13. doi: 10.2196/resprot.2184

Patel, V. L., \& Kushniruk, A. W. (1998). Interface design for health care environments: The role of cognitive science. In Proceedings of the AMIA Symposium (pp. 29-37).

Peddle, K. (2007). Telehealth in context: Socio-technical barriers to telehealth use in labrador, Canada. Computer Supported Cooperative Work (CSCW), 16(6), 595-614. doi: 10.1007/s10606-006-9030-3

Powell, R. E., Henstenburg, J. M., Cooper, G., Hollander, J. E., \& Rising, K. L. (2017). Patient perceptions of telehealth primary care video visits. Annals of Family Medicine, 15(3), 225-229.

Punchoojit, L., \& Hongwarittorrn, N. (2017). Usability studies on mobile user interface design patterns: A systematic literature review. Advances in Human-Computer Interaction. doi: 10.1155/2017/6787504

Redish, J. G. (2007). Expanding usability testing to evaluate complex systems. Journal of Usability Studies, 2(3), 102-111.

Rogers, Y., Preece, J., \& Sharp, H. (2007). Interaction design: Beyond human-computer interaction. West Sussex, UK: John Wiley \& Sons.

Roman, L. C., Ancker, J. S., Johnson, S. B., \& Senathirajah, Y. (2017). Navigation in the electronic health record: A review of the safety and usability literature. Journal of Biomedical Information, 67, 69-79. doi: 10.1016/j.jbi.2017.01.005

Sadeghi, P., Andreev, P., Benyoucef, M., Momtahan, K., \& Kuziemsky, C. (2014). Activity theory drive system analysis of complex healthcare processes. In Proceedings of the European Conference on Information Systems (ECIS). Israel.

Sandefer, R. H., Westra, B. L., Khairat, S. S., Pieczkiewicz, D. S., \& Speedie, S. M. (2015). Determinants of consumer eHealth information seeking behavior. In Proceedings of the AMIA Annual Symposium (pp. 1121-1129).

Sarkar, U., Karter, A. J., Liu, J. Y., Adler, N. E., Nguyen, R., Lopez, A., \& Schillinger, D. (2010). The literacy divide: Health literacy and the use of an internet-based patient portal in an integrated health system - Results from the diabetes study of northern California (DISTANCE). Journal of Health Communication, 15(Suppl 2), 183-196.

Schneider, T., Panzera, A. D., Couluris, M., Lindenberger, J., McDermott, R., \& Bryant, C. A. (2016). Engaging teens with asthma in designing a patient-centered mobile app 
to aid disease self-management. Telemedicine and E-Health, 22(2), 170-175.

Spinuzzi, C. (1999) Grappling with distributed usability: A cultural-historical examination of documentation genres over four decades. In Proceedings of the 17th annual International Conference on Computer Documentation (pp. 16-21). New Orleans.

St.Amant, K. (2017). Context, culture, and usability. Intercom, 63(7), 31-32.

St.Amant, K. (2019a). The cultural context for communicating care. Journal of Technical Writing and Communication, 49(4), 367-382.

St.Amant, K. (2019b). Usability in contexts of care. Intercom, 65(5/6), 35-36.

Tuckson, R. V., Edmunds, M., \& Hodgkins, M. L. (2017). Telehealth. The New England Journal of Telemedicine, 377, 1585-1592. doi: 10.1056/NEJMsr1503323

Turner, P., Kushniruk, A., \& Nohr, C. (2017). Are we there yet? Human factors knowledge and health information technology - The challenges of implementation and impact. Yearbook of Medical Informatics, 26(1), 84-91.

Vrazalic, L. (2003). Evaluating distributed usability: The role of user interfaces in an activity system. Australasian Journal of Information Systems, 11(1), 26-39.

Vygotsky, L. S. (1978). Mind in society: The development of higher psychological processes. Cambridge, MA: Harvard University Press.

Wang, X., Zhang, Z., Zhao, J., \& Shi, Y. (2019). Impact of telemedicine on healthcare service system considering patients' choice. Discrete Dynamics in Nature and Society. doi: $10.1155 / 2019 / 7642176$

Wax, C. M. (2019, January 29). What is "healthcare?" Medical Economics. Retrieved from https://www.medicaleconomics.com/view/what-healthcare

Whitten, P. (2006). Telemedicine: Communication technologies that revolutionize healthcare services. Generations, 30(2), 20-24.

Wilson, E. V., \& Lankton, N. K. (2004). Modeling patients' acceptance of providerdelivered e-Health. Journal of the American Medical Informatics Association (JAMIA), 11(4), 241-248.

Wilson, M. (2009). Readability and patient education materials used for low-income populations. Clinical Nurse Specialist, 23(1), 33-40.

Wilson, T. D. (2008). Activity theory and Information Seeking. Annual Review of Information Science and Technology, 42(1), 119-161.

Wiser, F., Durst, C., \& Wickramasinghe, N. (2017). Analyzing collaborative patient care solutions using activity theory. In Proceedings of the 50th Hawaii International Conference on System Sciences (pp. 901-909).

Yen, P. Y., \& Bakken, S. (2011). Review of health information technology usability study methodologies. Journal of the American Medical Informatics Association, 19(3), 413-422.

Zhang, J., \& Waljiac, M. F., (2011). TURF: Toward a unified framework of EHR usability. Journal of Biomedical Informatics, 44(6), 1056-1067. 